Astrophysics 63, 440-446 (2020)

doi: 10.1007/s10511-020-09648-x

(C) 2020. Springer Science+Business Media, LLC. Translated from Astrofizika (August 2020).

\title{
ON THE ORIGIN OF OPTICAL RADIATION DURING THE IMPULSIVE PHASE OF FLARES ON dMe STARS. II. CONTINUUM AND LINE RADIATION
}

\author{
E. S. Morchenko \\ Physics Faculty, M. V. Lomonosov Moscow State University, Moscow, Russia; \\ e-mail: morchenko@physics.msu.ru \\ Original article submitted December 16, 2019; accepted for publication June 24, 2020
}

It is argued that not only the blue (at the brightness maximum) but also the red (in the slow decay phase) components of the optical continuum of powerful flares on dMe stars are formed near the photosphere. The possible appearance of HeI lines in the relaxation zones for the plasma to a state of thermal equilibrium (as a result of a rise in electron temperature owing to elastic collisions of electrons with atoms and ions) is noted (for sufficiently high speeds of a non-stationary chromospheric shock wave propagating toward the photosphere of the Sun and stars). A scheme is proposed for the positioning of the "layers" of plasma responsible for the generation of radiation in the white light continuum during the impulsive phase of powerful stellar flares.

Keywords: red dwarf stars: flares: the flare models: optical radiation

1. Introduction. This article completes the discussion begun by the author in Ref. 1.1

2. Radiation from near-photospheric layers. Belova and Bychkov [2] assume that "black body radiation that appears at times during flares" ... "comes from a photosphere heated by a flux of suprathermal particles;" "a hot spot with a temperature of $10^{4} \div 2 \cdot 10^{4} \mathrm{~K}$ may be formed in it" [2].

In these assertions the authors [2] do not take the following into account. By definition, the visibility of flares indicates the existence of unperturbed layers of a star's atmosphere. In the method of Ref. 3 for estimating the area $s$ of the source of a (quasi-black-body) "blue" continuum of powerful flares on dMe stars (the brightness maximum) in the approximation of an absolute black body (used, for example, in Ref. 4) these layers are identified with the photosphere of a red dwarf in a quiescent state, and the photosphere radiation field is assume to be Planckian.2 In addition, recent

\footnotetext{
${ }^{1}$ Here (as well as in [1]), the "blue" continuum is considered as part of the flare continuum with a sufficiently large optical depth beyond the Balmer jump. This opacity is a necessary condition [3] for explaining the blue color of powerful stellar flares at the brightness maximum. From a physical standpoint, the "blue" continuum is the same as the "[quasi-]black-body component" in terminology Kowalski et al. [12] (including the contribution in the $U$ band), as it follows from Fig. 2 in [10] or identical Fig. 1 in [19].

${ }^{2}$ We note that the values of $s$ estimated in Ref. 4 are reduced by a factor of 2 . This is because of an imprecision in Eq. (3); the luminosity of a compact formation radiating according to the Planck law is equal to [3] $2 \pi s B_{\nu}\left(T_{b b}\right)$, where $T_{b b}$ is the temperature of an absolute black body corresponding to the spectrum of a flare in the wavelength $\lambda$ range from 4000 to $4800 \AA$.
} 
observations [5] of the flare activity of Proxima Cen (dM5.5e) indicate that the molecular absorption spectra of the atmosphere of the red dwarf (the reversing layer) "are essentially independent of the flare events." In turn, Honda et al. [6] did not detect changes in the molecular bands during the time of a flare on EV Lac (dM4.5e).

The quasi-black-body shape of the blue continuum of powerful flares on dMe stars at the brightness maximum is indicated by the existence of continuum optical radiation on the long-wavelength (red) side from the $\mathrm{H}_{\beta}$ line, this radiation does not correspond to a Planck function with $T_{b b} \sim 10^{4} \mathrm{~K}$ (see Fig. 8a in the article by Kowalski et al. [7]).

Such radiation originates in deeper, less heated near-photospheric layers. Here an important role in generating the quasi-black-body (a small Balmer jump) "red" component which predominates in the optical continuum of flares during the slow decay phase (Fig. 31 in Ref. 7; the wavelengths excluding "the conundruum $=$ conundrum + continuum") is played [3, 8] by the negative hydrogen ion (for $T_{b b}$ approximately $<8000 \mathrm{~K}$ the donors of free electrons are metals; see Ref. 9, §6.5). In terms of this interpretation, the "relationship" [7] of the blue and red components of the continuous radiation of flares, as well as the "relatively more important" [7] contribution of the red component "to the energy budget during the later stage of the slow decay phase" [7] seems entirely natural.

Thus, not only the blue but also the red components of the optical continuum of powerful flares on dMe stars are formed near the photosphere [3], while the assertions of the authors of Ref. 2 (and, as a whole, the tendency to "invoke" "layers" of the photospheres of dMe stars for interpretation of observations of flares) conflict with observational data.

The rapid change in the optical continuum of near-photospheric layers during the impulsive phase of flares makes it difficult to study gas dynamic processes taking place in higher lying layers of the chromosphere since the radiation field of the near-photospheric gas does influence [10, 11] the ionization state of these layers. Thus, for $T_{b b}=1.1 \mathrm{eV}\left(\approx 1.3 \cdot 10^{4} \mathrm{~K}\right)$, the electron density $n_{e}=10^{14} \mathrm{~cm}^{-3}$, the electron temperature $T_{e}=1 \mathrm{eV}$, the rate of photoionization by the diluted (dilution factor $\mathrm{W}=0.5$ ) Planckian radiation field from the second level of the hydrogen atom is $\approx 4.6$ times greater ( [11], Table 3.2) than the corresponding electron impact ionization rate (results obtained for the brightness maximum of a powerful flare; the approximation for an absolute black body used here is correct because the quasi-black-body "blue" continuum at the peak of the flare makes a significant contribution to the radiation including in the $U$ band; see section 3.3 in the paper by Kowalski et al. [12]). At the same time, the rise in $T_{e}$ behind the front of the stationary radiative shock wave owing to elastic collisions of electrons with atoms and ions for $T_{a i} \gg T_{e}$ [10] (as in the calculations of Ref. 2) assumes a moderate degree of ionization of the unperturbed chromospheric gas (ahead of the front); here $T_{a i}$ is the atom-ion temperature of the plasma 3

3. Neutral helium lines. For high velocities $v_{s h}$ of the non-stationary chromospheric shock wave

\footnotetext{
${ }^{3}$ We note that: (a) the authors of Ref. 2, as opposed to Ref. 10, have incorrectly calculated the probability of escape $\theta_{12}$ of a resonance photon beyond the confines of the plasma without scattering (substituting the Doppler optical depth in an expression obtained for a Holtsmark profile); (b) in Ref. 10, in the case of emergence of a photon from the center of a plane layer $\theta_{12}^{c} \approx 0.6 \bar{\theta}_{12}<\bar{\theta}_{12}$, where $\bar{\theta}_{12}$ is the value of $\theta_{12}$ averaged over the layer (Eq. (55) in Ref. 10).
} 
propagating in a partially ionized plasma in the direction of the photosphere, this increase in the electron temperature makes it possible to create conditions for ionization of helium atoms (HeI) and excitation of their discrete levels by electron impact (radiative cooling is substantially nonstationary [2], so the degree of ionization and the excitation state of the atoms are determined not only by the current value of $T_{e}$ ); this is a known effect [13] in the theory of stationary shock waves with emission. In this case the subsequent de-excitation by allowed spontaneous transitions can ensure [1] the appearance and enhancement of HeI lines in the spectra of powerful solar and stellar flares (e.g., lines with $\lambda=10830 \AA$ [14]). We recall, however (see Ref. 15), that $v_{s h}$ is increased by raising the energy flux $F_{0}$ in the beam of accelerated electrons.

We emphasize that, because of the rapid radiative cooling of the dense gas behind the shock front (e.g., Ref. 1) the situation described above: (a) corresponds to zones in which the plasma relaxes to a state of thermal equilibrium for a certain range of velocities $v_{s h}$ (this effect can be illustrated in a model of a "set" of stationary shocks with emission [2]); (b) is detected for heating pulses that partially overlap in time and act on different segments of the upper chromosphere of the Sun and dMe stars.

4. $\mathrm{H}_{\alpha}$ lines with "blue" asymmetry in the wings. It is known that in the spectra of some flares on dMe stars long-lived asymmetric profiles of the $\mathrm{H}_{\alpha}$ lines are observed with enhanced intensity in the blue wing rather then the red (e.g., Refs. 16 and 6). Honda et al. [6] noted a possible relationship between the formation of radiation of this sort and the presence in the flare atmosphere of a red dwarf of cold $\left(T \sim 10^{4} \mathrm{~K}[6]\right.$ ) gas. At the same time, the existence of broad wings in $\mathrm{H}_{\alpha}$ (Fig. 7, Ref. 16) is evidence (Eason et al. [16], Fig. 9; an approximation of part of the red wing of the line by a Stark profile) of a fairly high gas density that is responsible for their formation. (We note that the value of $\log n_{e}$ is found in Ref. 16 without accounting for the significant optical depth of $\mathrm{H}_{\alpha}$ in the core (the characteristic "dip" in the center) that is indicative of possible opacity of this line even in the wings.) Using $\Delta \lambda_{D}$ (the Doppler half width) and $\log n_{e}$ found in Ref. 16, Morchenko et al. [1, 10] showed (section 5.3 in [10]; introduction to [1]) that the $\mathrm{H}_{\alpha}$ profile in the observations [16] is approximately described by a model with a "Doppler core" and "Stark-broadened wings."

Honda et al. [6] also give possible explanations of the nature of the "blue" asymmetry in the wings of $\mathrm{H}_{\alpha}$ known from the physics of solar flares. These explanations, however, are pointed out as insufficient by the authors [6].

Given these remarks, there is some interest in interpreting the nature of the blue asymmetry in the wings of $\mathrm{H}_{\alpha}$ based on the existence of a velocity field of gas in a chromospheric condensation and in thermal relaxation zones [17]. According to this point of view, "the type of asymmetry is determined by the sign of the velocity gradient in that region where the intensity of the central part of the line is formed" (a red asymmetry corresponds to expansion of this region and a blue, to its compression [17]); here the resulting line profile shows up [17] as a superposition of profiles that take into account the non-uniformity of the physical parameters of the beams of accelerated electrons (see Fig. 3 of Ref. 17). This interpretation is favored by the absence of a significant Doppler shift in the center of the $\mathrm{H}_{\alpha}$ line in observational data [6, 16].

5. Emission in the "white" light continuum. The author, therefore, proposes the following 
scheme for the locations of the plasma "layers" responsible for the continuum (in white light) and line radiation during the impulsive phase of powerful flares on dMe stars:

(a) a gas that is transparent in the Balmer continuum is formed mainly in a dynamically inhomogeneous chromospheric condensation (the optical depth at a wavelength of $4170 \AA, \tau_{4170} \ll 1$, as in Refs. 18 and 19) and also in thermal relaxations zones 4 and in the region ahead of a non-stationary chromospheric shock wave (the precursor and heating by high-energy, $E \gg E_{10}\left(E_{10}\right.$ is the cutoff on the low energy side), electrons from a beam with a decaying power-law spectrum). This point of view agrees qualitatively (for the chromospheric condensations and the thermal relaxations zones - there is a substantial Balmer jump) with the results of an analysis of the spectra of the flare YZ CMi (dM4.5e) in the NUV wavelength range (from 3350 to $\lambda<3646 \AA$ A) by Kowalski et al. [12] (Fig. 1c in [12], the response of the chromosphere of the red dwarf to heating by a beam of non-thermal electrons with an energy flux of $F_{0}=10^{11} \mathrm{erg} / \mathrm{cm}^{2} \mathrm{~s}$ );

(b) the quasi-black-body "blue" (at the maximum brightness of powerful flares, $T_{b b} \sim 10^{4} \mathrm{~K}$ ), $\lambda$ from 4000 to $4800 \AA$, and red (in the slow decay phase) components of the optical continuum arise near the photosphere (Grinin and Sobolev [3]);

(c) the HI emission lines are mainly localized in the chromospheric condensation and zones of thermal relaxation. Here the populations of the atomic levels and the degree of ionization of the plasma are: (a) close to the equilibrium values in a chromospheric condensation with sufficient geometric thicknes:5 (Kowalski and Allred [20], Morchenko et al. 11, 10]) and in the region where the blue continuum is formed (at the brightness maximum of powerful flares) [3, 19]; (b) differ sharply [20] from the equilibrium values in the thermal relaxation zones behind the front of the non-stationary chromospheric shock wave (according to the general rule the deviations for the populations of the atomic levels $\nu_{k}$ decrease with increasing principal quantum number $k$ ). The gas radiating behind the front of the non-stationary chromospheric shock wave is stable with respect to radiative cooling (this conclusion follows from the calculations of Ref. 2). The dense plasma of the chromospheric condensation, which is near a state of complete thermalization, ensures (see the review [21]) the gently sloping and inverse Balmer decrements that are characteristic (e.g., [22]) of the brightness maxima of flares on red dwarfs.

The following observational data confirm the validity of this scheme:

(a) the time "evolution" of the Balmer continuum closely follows the corresponding time variations in the atomic hydrogen Balmer lines (Kowalski et al. [12], bottom part of Fig. 1d). This fact indicates some relationship between their formation;

(b) "the Balmer continuum manifests a slow decay ..., which is typical of emission in the Balmer series" lines of the hydrogen atom in the spectra of flares [12, 22];

(c) during the time a flare is decaying, the emission region in the Balmer continuum is "always considerably ( $\sim 3-16$ times) greater" [12] than the region occupied by the component with $T_{b b} \sim$ $10^{4} \mathrm{~K}$. This result can be interpreted as, on one hand, an increase in the geometric thickness of the

\footnotetext{
${ }^{4}$ With a geometric thickness that is considerably less than the thickness of the chromospheric condensation toward the end of the heating.

${ }^{5}$ The non-stationary shock front has substantially "overtaken" the temperature jump.
} 
region between the temperature jump and the front of the non-stationary shock wave with emission (during the time of impulsive heating the thermal relaxation zone "travels" into a region of ever higher values of the Lagrangiane coordinate $\xi$ (see Fig. 3 in Ref. 18), while the temperature jump is essentially motionless relative to the shock front) and, on the other hand, as a transition of part of the plasma in the near-photospheric layers into a state in which the spectrum of the radiation is transparent beyond the Balmer jump;

(d) the time evolution of the radiation in the $U$ band differs ( [12], Fig. 1d) from the behavior of the Balmer continuum, with the largest deviations corresponding [12] to the peaks in the light curve (e.g., at $t \sim 130 \mathrm{~min}$ ), when the flare spectrum is dominated [4, 12] by the quasi-black-body "blue" component of the optical continuum. Thus, these components of the radiation in white light are formed in different regions of the chromosphere;

(e) during the slow decay phase of flares, in the region beyond the Balmer jump a larger range of wavelengths is "occupied" by the "red" component of the optical continuum (Fig. 31 in [7]). This result is explained by the fact that as the hydrogen plasma undergoes a transition into a state close to LTE (the quasi-black-body spectrum), the red part of the spectrum thermalizes initially (Morchenko et al. [10], Fig. 2), and then the blue.

Furthermore, the increase in the energy flux $F_{0}$ for fixed $E_{10}$ and spectral index (indices) of the non-thermal electrons leads to a reduction in the "evolution" time of the chromospheric condensation and the thermal relaxation zones. From a physical standpoint this happens because (a) the higher values of $F_{0}$ correspond to higher velocities $v_{s h}$, so that the shock front "moves" into the depth of the chromosphere of dMe stars more rapidly; (b) large $v_{s h}$ causes stronger compression of the gas behind the non-stationary shock front (for an ideal monatomic gas with a constant specific heat the limiting compression is 4 ), and, thereby, more rapid (e.g., Ref. 1) radiative cooling. Hence, the use of extremely high, $F_{0}=10^{13} \mathrm{erg} / \mathrm{cm}^{2} \mathrm{~s}$ (Kowalski et al. [23]), energy fluxes in gas dynamic calculations of the type in Ref. 18 lead to a shortening of the "lifetime" of the Balmer lines, which is inconsistent with the need to interpret their long-term emission [12, 22] in the spectra of flares.

We note that in the proposed scheme, the gas generating the quasi-black-body radiation (at the maximum brightness of powerful flares) lies much deeper than the plasma responsible for the line spectrum and the spectrum transparent in the Balmer continuum (a positive gradient of the density in the perturbed chromosphere of a red dwarf). In addition, this sort of scheme starts with moderately high, from $\sim 10^{11} \mathrm{erg} / \mathrm{cm}^{2} \mathrm{~s}$ to $\sim 10^{12} \mathrm{erg} / \mathrm{cm}^{2} \mathrm{~s} F_{0}$ (the return current problem), and is consistent (Tsap et al. [24]) with the standard model of solar flares, since it does not assume the existence (see the references in [24]) of dense $\left(n_{e}>10^{12} \mathrm{~cm}^{-3}\right)$ flare coronal loops 6

6. Conclusion. In the author's opinion, a theoretical check of the agreement between this scheme and observations of flares will involve: (a) a study of mechanisms for accelerating particles to high energies in the model [3] and (b) a study of the effect of the radiation field of the chromospheric plasma (the gas with $T \sim 10^{7} \mathrm{~K}[18$, the thermal wave; the thermal relaxation zones; the gas ahead of the non-stationary shock wave) on the deep (near-photospheric) layers of flare (dMe) stars.

\footnotetext{
${ }^{6}$ In the calculations [18] the contribution of soft x-rays to heating the dense layers of the chromosphere $\left(\xi \sim 10^{21} \mathrm{~cm}^{-2}[18]\right)$ is accounted for in the heating function $P_{e}(\xi)$ by taking the coefficient $\beta=1$.
} 


\section{References}

1. E. S. Morchenko, Astrophysics 63, 91 (2020) http://astro.asj-oa.am/4283/1/Morchenko.pdf. arXiv: 2004.14508 [astro-ph. SR].

2. O. M. Belova and K. V. Bychkov, Astrophysics 62, 234 (2019); 61, 224 (2018).

3. V. P. Grinin and V. V. Sobolev, Astrophysics 13, 348 (1977). doi: 10.1007/BF01006610 [http://astro.asj-oa.am/3793/1/587.pdf]

4. B. E. Zhilyaev, Ya. O. Romanyuk, O. A. Svyatogorov, et al., Astron. Astrophys. 465, 235 (2007).

5. Ya. V. Pavlenko, A. Suárez Mascareño, M. R. Zapatero Osorio, et al., Astron. Astrophys. 626, A111 (2019).

6. S. Honda, Y. Notsu, K. Namekata, et al., Publ. Astron. Soc. Japan 70, 62 (2018).

7. A. F. Kowalski, S. L. Hawley, J. P. Wisniewski, et al., Astrophys. J. Suppl. Ser. 207, 15 (2013).

8. V. P. Grinin, private communication (2015).

9. V. V. Sobolev, A Course in Theoretical Astrophysics, NASA Technical Translation TT F-531, Washington, DC (1969).

10. E. Morchenko, K. Bychkov, and M. Livshits, Astrophys. Space Sci. 357, 119 (2015). arXiv: 1504.02749.

11. E. S. Morchenko, Candidate's Dissertation, Phys. Math. Sci., M. V. Lomonosov Moscow State University (2017).

12. A. F. Kowalski, S. L. Hawley, J. A. Holtzman, et al., Astrophys. J. Lett. 714, L98 (2010).

13. A. A. Korovyakovskaya, Astrophysics 8, 148 (1972). doi: 10.1007/BF01002164

14. S. J. Schmidt, A. F. Kowalski, S. L. Hawley, et al., Astrophys. J. 745, 14 (2012).

15. M. K. Druett and V. V. Zharkova, Astron. Astrophys. 610, A68 (2018).

16. E. L. E. Eason, M. S. Giampapa, R. R. Radick, et al., Astron. J. 104, 1161 (1992).

17. N. D. Kostyuk, Soviet Astron. 20, 206 (1976).

18. M. M. Katsova, A. G. Kosovichev, and M. A. Livshits, Astrophysics 17, 156 (1981).

19. E. S. Morchenko, Astrophysics 59, 475 (2016). arXiv: 1710.08008 [astro-ph. SR].

20. A. F. Kowalski and J. C. Allred, Astrophys. J. 852, 61 (2018).

21. V. P. Grinin, Astrophysics 20, 190 (1984). doi: 10.1007/BF01005834 [http://astro.asj-oa.am/2791/]

22. R. E. Gershberg, Astrophysics 13, 310 (1977). doi: 10.1007/BF01001231

23. A. F. Kowalski, S. L. Hawley, M. Carlsson, et al., Solar Phys. 290, 3487 (2015).

24. Yu. T. Tsap, A. V. Stepanov, Yu. G. Kopylova, et al., in: Solar and Sun-Earth Physics (2019); A. V. Stepanov and Yu. A. Nagovitsyn, eds., Pulkovo Observatory, RAS, St. Petersburg, p. 431 (2019) (http://www.gaoran.ru/russian/solphys/2019/book/gao2019.pdf). 